\title{
THE DETERMINATION OF ERRORS IN POLARIZED NEUTRON DIFFRACTOMETRY
}

\author{
H KENDRICK* \\ Dept. of Nuclear Engineering, The University of Michlgan, Ann Arbor, Michigan, $U S A$ \\ $S$ A WERNER \\ Scientıfic Laboratory, Ford Motor Company, Dearborn, Michigan, U.S.A \\ and \\ A. ARROTT \\ Sctentific Laboratory, Ford Motor Company, Dearborn, Michigan, US A \\ Dept of Nuclear Engineering, The Unversity of Michigan, Ann Arbor, Michigan, US A
}

Recelved 30 August 1968

The polarized neutron method of determining the magnetıc form factor of magnetic materials is examined in detail with special attention given to the way in which statistical errors are propagated Because of the nonlinear relation between the polarizing efficiency of a crystal and the magnetic scattering length, the usual methods of linear error theory will not work. However, this

\section{Introduction}

The primary use of a polarized neutron spectrometer has been in the form factor determination of ferromagnets and some ferrimagnets and antiferromagnets ${ }^{1-6}$ ). The purpose of this paper is to examine this method of measuring magnetic scattering amplitudes, to discuss the importance of the various correction factors and to show how the statistical errors are propagated.

In section 2 the familiar formula for the flipping ratio or polarization ratio is introduced with emphasis on the assumptions required to derive it from the general expression for the cross section. The experimental arrangement of the polarized neutron spectrometer is described in section 3. The Shim Ratıo Method for the measurement of the polarization of the beam $P$ and the flupping efficiency $\varepsilon$ of the $\mathrm{rf}$ coil is examined ${ }^{2}$ ). It is pointed out that a measurement, additional to those usually made, is the key to a reliable determination of $P$ and $\varepsilon$. This extra measurement is of the intensity scattered from the analyzing crystal with both the flipper on and the depolarizing shım in the beam. Thıs gives a sensitive measurement of the depolarizing efficiency of the shim and ensures a consistent set of readings. In section 4 the expression for the experimentally measured flipping ratio is derived and the assumptions required to arrive at the simple expression of section 2 are pointed out. In section 5 expressions for the statistical errors are developed and tabulated

* Present address: John Jay Hopkins Laboratory of Pure and Applıed Science, Gulf General Atomic, San Diego, Calıfornı. difficulty can be circumvented by using the nonlinear formulas directly. The statistical error analysis is applied to the practical problem of how long one should count on a particular Bragg peak. This tıme will depend on the ratio of the magnetic to the nuclear scattering lengths, $p / b$, and on the accuracy with which the beam polarizations and flipping efficiencies have been determined

for values of $P$ and $\varepsilon$ of 1.0 and 0.9 . A typical experimental result is taken in order to illustrate how to assess the error in a practical case. In section 6 we discuss four of the principal systematic errors which can occur, (a) extınction; (b) drift of the flipper efficiency; (c) spin-flip scattering cross sections, and (d) depolarization in the specimen. Section 7 uses the statistical error analysis to calculate counting-time-ratios for the case when it is desired to have equal error contributions from all the intensity measurements involved in determining the form factor. The principal results and conclusions are summarized in section 8 .

\section{Polarized neutron scattering cross section}

In the Born approximation, an elastic scattering cross section involves the Fourier transform of the interaction potential. Classically the energy of interaction of a point-dipole $\boldsymbol{\mu}$ with a magnetic field $\boldsymbol{B}$ is $\boldsymbol{\mu} \cdot \boldsymbol{B}$. In quantum mechanics $\boldsymbol{\mu}$ and $\boldsymbol{B}$ are operators. We take the operator corresponding to the dipole moment of the neutron as the Pauli spin matrices $(\sigma)$ with the corresponding neutron spin wave functions $\left(\begin{array}{l}1 \\ 0\end{array}\right)$ and $\left(\begin{array}{l}0 \\ 1\end{array}\right)$. The expectation value of the operator $B$ is the actual magnetic induction field appearing in Maxwell's equations. We use the same symbol $B$ to represent this expectation value. When the wave vector of a Fourier component of the magnetic induction is the same as that of a Fourier component of the mass density, then there is interference between the nuclear and magnetic elastic neutron scattering. The elastic Bragg scattering cross sections which a magnetic material presents to 
an incident beam polarized to a degree $P_{1}$ along a direction $\hat{\boldsymbol{P}}$ are given by ${ }^{7}$ ):

$$
\begin{aligned}
& \mathrm{d} \sigma_{\uparrow \uparrow} / \mathrm{d} \Omega \propto\left|V_{g}+\mu_{\mathrm{n}} g_{\mathrm{N}} \boldsymbol{B}_{g} \cdot \hat{\boldsymbol{P}}\right|^{2} \cdot \frac{1}{2}\left(1+P_{1}\right), \\
& \mathrm{d} \sigma_{\downarrow \downarrow} / \mathrm{d} \Omega \propto\left|V_{g}-\mu_{\mathrm{n}} g_{\mathrm{N}} \boldsymbol{B}_{g} \cdot \hat{\boldsymbol{P}}\right|^{2} \cdot \frac{1}{2}\left(1-P_{1}\right), \\
& \mathrm{d} \sigma_{\uparrow \downarrow} / \mathrm{d} \Omega \propto\left\{\left|\mu_{\mathrm{n}} g_{\mathrm{N}} \boldsymbol{B}_{g}\right|^{2}-\left|\mu_{\mathrm{n}} g_{\mathrm{N}} \boldsymbol{B}_{g} \cdot \hat{\boldsymbol{P}}\right|^{2}\right\} \cdot \frac{1}{2}\left(1+P_{1}\right), \\
& \mathrm{d} \sigma_{\downarrow \uparrow} / \mathrm{d} \Omega \propto\left\{\left|\mu_{\mathrm{n}} g_{\mathrm{N}} \boldsymbol{B}_{g}\right|^{2}-\left|\mu_{\mathrm{n}} g_{\mathrm{N}} \boldsymbol{B} g \cdot \hat{\boldsymbol{P}}\right|^{2}\right\} \cdot \frac{1}{2}\left(1-P_{1}\right),
\end{aligned}
$$

where

$$
-1 \leqq P_{1} \leqq+1 .
$$

$V_{g}$ and $B_{g}$ are the Fourier components of the neutronnuclear interaction potential $V(\boldsymbol{R})$ and the magnetic induction $\boldsymbol{B}(\boldsymbol{R})$ corresponding to the reciprocal lattice vector $g$ respectively. $\mu_{\mathrm{n}} g_{\mathrm{N}}$ is the magnetic moment of the neutron. The arrow subscripts on the cross sections denote the initial and final spin directions of the neutron with respect to the direc'ion $\hat{\boldsymbol{P}}$. It is evident that components of $\boldsymbol{B}_{\boldsymbol{g}}$ not parallel to $\hat{\boldsymbol{P}}$ contribute to spin-fllp processes.

The Fourier components of the magnetic induction and the magnetization are related through Maxwell's equations by

$$
B_{g}=4 \pi M_{g}-4 \pi g\left(g \cdot M_{g}\right) /|g|^{2},
$$

where $\boldsymbol{M}_{\boldsymbol{g}}$ is the Fourier component of the magnetızation $M(R)$.

In a polarized beam experiment in which the polarization of the incident beam $\boldsymbol{P}_{1}$ is reversed, the only term which is affected is the interference term between the magnetic and nuclear scattering. Consequently, the change of intensity observed in such an experiment is caused only by that part of the Fourier component of the magnetization which is both perpendicular to $g$ and parallel to the polarization direction. In general, therefore, this experiment does not measure the true form factor (the normalized Fourier transform of the magnetic moment density) which is really a vector quantity.

It is well known that, for polarized neutron work, ferromagnetic crystals must be saturated; that is, the net magnetization of all the domains must be aligned. The polarization of the incident beam is also aligned by the external field which is applied to the sample. We will call this the $+z$-direction. The magnetic part of the interference term, therefore, contains only $B_{g z}$, whereas the pure magnetic term is proportional to $\left|\boldsymbol{B}_{g}\right|^{2}$. The existence of the components $B_{g x}$ and $B_{g y}$ has not been precluded by the assumption of a net (bulk) magnetization. If the magnetization is periodic with the lattice, the $B_{g x}$ and $B_{g y}$ cannot be determined simply by observation of the flipping ratio $R$ :

$$
R=\frac{V_{g}^{2}+\left|\mu_{\mathrm{n}} g_{\mathrm{N}} \boldsymbol{B}_{g}\right|^{2}+2 P_{1} \mu_{\mathrm{n}} g_{\mathrm{N}} V_{g} B_{g z}}{V_{g}^{2}+\left|\mu_{\mathrm{n}} g_{\mathrm{N}} \boldsymbol{B}_{g}\right|^{2}-2 P_{1} \mu_{\mathrm{n}} g_{\mathrm{N}} V_{g} B_{g z}}
$$

We again note that $B_{g x}$ and $B_{g y}$ contribute to spin-flip processes only. In the special case that $B_{g x}$ and $B_{g y}$ represent a normal helical wave propagating in the $z$-direction, then $\left(B_{g x}^{2}+B_{g y}^{2}\right)$ could be observed directly from $R$ by applying the external field along the scatterıng vector $\boldsymbol{K}=\boldsymbol{g}$. In this case $\boldsymbol{B}_{\boldsymbol{g z}}$ is zero [eq. (5)] and the polarization effect of normal helical waves was pointed out by Overhauser ${ }^{8}$ ) although it has not yet been observed experimentally in elastic scattering. Otherwise the polarization of the scattered beam must be analyzed.

If we make the simplifying assumption that all the Fourier components of the magnetization are parallel or antiparallel to the single direction picked by an external field applied perpendicular to $g$, then there is no spin-flip scattering. This assumption that the magnetization direction does not vary across the atom is in accordance with the rigid spin model ${ }^{9}$ ). If we also assume that all atoms are equivalent, then the measured flipping ratı can be written

$$
R=\left(b^{2}+p^{2}+2 P b p\right) /\left(b^{2}+p^{2}-2 \varepsilon P b p\right),
$$

where $P=P_{1}$. The nuclear scattering length $b$ is given by

$$
b=\left\{M_{\mathrm{n}} /\left(2 \pi \hbar^{2}\right)\right\} V_{g} V_{\text {cell }} \quad\left[10^{-12} \mathrm{~cm}\right],
$$

where $M_{\mathrm{n}}$ is the neutron mass, and $V_{\text {cell }}$ is the volume of the unit cell. The magnetic scattering length $p(g)$ is given by

$$
p(g)=0.2695 \mu_{0} f(g) \quad\left[10^{-12} \mathrm{~cm}\right],
$$

where $\mu_{0}$ is the magnetic moment per atom in Bohr magnetons and $f(g)$ is the form factor of the atom. The quantity $\varepsilon$ is defined as the efficiency with which a flipper turns the incident beam polarization from $P$ along the $+z$-axis to $-P$ along the $+z$-axis. Eq. (7) is the simple expression for the flipping ratio which is commonly used for ferromagnets ${ }^{10}$ ).

\section{Measurement of beam polarization and flipper efficiency}

A schematic diagram of the experimental arrangement ${ }^{1,7}$ ) used to measure magnetic form factors is shown in fig. 1. Magnetic fields of about $3000 \mathrm{G}$ are applied to the $\mathrm{Co}(8 \% \mathrm{Fe})$ monochromator and analyzer crystals and a guide field of about $150 \mathrm{G}$ is maintained 


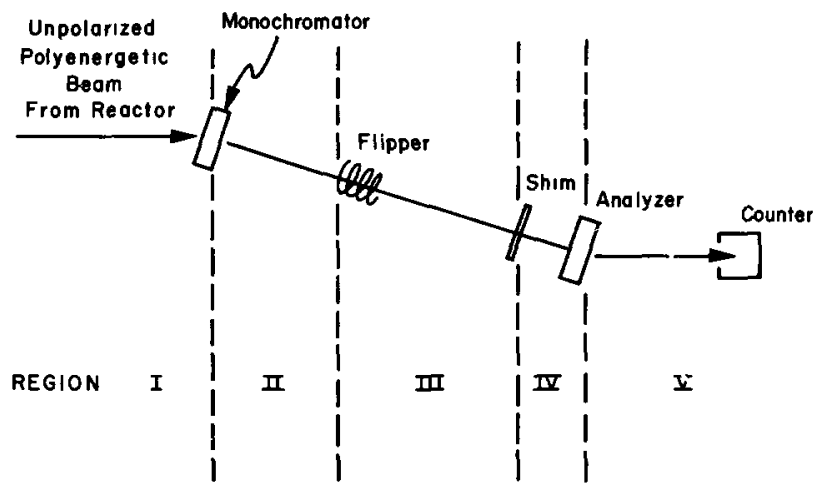

EXPERIMENTAL ARRANGEMENT

Fig 1 Schematic drawing of a polarized neutron experıment.

in the region between the monochromator and the shim which is about 18 " from the analyzer. All fields have the same sense. The flipper is a coil placed axially along or across the beam and supplied with rf power to provide a small oscillating field which is perpendicular to the guide field.

In the analysis that follows we make the simplifying assumption that the guide field causes no reorientation of any neutron spin. In other words spin-flip occurs in regıon II in the monochromator only, in region III only when the flipper is turned on, in region IV only when the shim is inserted, in region $V$ in the analyzer only. This assumption can be checked experimentally by observing the intensity scattered from the analyzer as various guide field parameters are changed. These parameters may be, for example, the distances between the ends of the guide field and the monochromator and analyzer, or the relative positions of the permanent magnets used to produce the guide field.

The $+z$-direction is defined by the applied magnetic field whether it 1 s the monochromator field, the guide field or the analyzer field. The $+z$ - and $-z$-durections are denoted by subscripts $\uparrow$ and ${ }_{\downarrow}$ respectively. Superscripts denote either a region (I, II, III, IV, V) or a crystal, monochromator (M), analyzer (A), sample (T). Thus, for example, $\mathrm{N}_{\uparrow}^{\mathrm{III}}$ is the number of neutrons in the $+\frac{1}{2}$ state in region III; and $\sigma_{\downarrow}^{\mathrm{A}}$ is the cross section for a neutron incident in the $-\frac{1}{2}$ state to leave the analyzer in the $+\frac{1}{2}$ state. The probability that a neutron spin is reoriented by $180^{\circ}$ in the flipper is defined as $f$ where we have assumed

$$
f_{\uparrow \downarrow}=f_{\downarrow \uparrow}=f .
$$

A similar quantity $q$ is defined for the shim. The polarization of the neutron beam $\mathrm{m}$, say, region III is defined to be

$$
P^{\mathrm{III}} \equiv\left(N_{\uparrow}^{\mathrm{III}}-N_{\downarrow}^{\mathrm{III}}\right) /\left(N_{\uparrow}^{\mathrm{III}}+N_{\downarrow}^{\mathrm{III}}\right) .
$$

We also define the polarizing efficiency of, say, the analyzing crystal to be the polarization of the beam scattered by the analyzer when the incident beam is unpolarized $\left(N_{\uparrow}=N_{\downarrow}\right)$. Thus

$$
P(\mathrm{~A})=\left[\left(\sigma_{\uparrow \uparrow}^{\mathrm{A}}+\sigma_{\downarrow \uparrow}^{\mathrm{A}}\right)-\left(\sigma_{\downarrow \downarrow}^{\mathrm{A}}+\sigma_{\uparrow \downarrow}^{\mathrm{A}}\right)\right] / \sigma^{\mathrm{A}},
$$

where

$$
\sigma_{\mathfrak{\sharp}}^{\mathrm{A}} \equiv \sigma_{\uparrow \uparrow}^{\mathrm{A}}+\sigma_{\uparrow \downarrow}^{\mathrm{A}}+\sigma_{\downarrow \uparrow}^{\mathrm{A}}+\sigma_{\downarrow \downarrow}^{\mathrm{A}}{ }^{\top \uparrow} .
$$

Note that, by definition

$$
P^{\mathrm{II}}=P(\mathrm{M})^{\top}
$$

We can now write down the number of neutrons of each polarization state in each region with the help of these definitions, and these results are given in table 1.

We now consider the intensity scattered from the analyzer under four conditions: (1) Flipper off, Shım out; (2) Flipper off, Shim in; (3) Flipper on, Shim out; (4) Flipper on, Shım in. We denote these intensities by $I_{J}(j=1,2,3,4)$ and note that

$$
I_{J}=N_{\uparrow}^{\mathrm{V}}+N_{\downarrow}^{\mathrm{V}} \text {. }
$$

These four measured intensitıes are conveniently defined in terms of three ratios:

$$
\begin{array}{r}
\text { Shim ratio }(S) \equiv I_{1} / I_{2} \\
\text { Flipping ratio }(R)=I_{1} / I_{3} \\
\text { Special flipping ratio }\left(R_{\mathrm{s}}\right) \equiv I_{2} / I_{4}
\end{array}
$$

Expressions for these ratios are shown in the second column of table 2 . We note the similarity between the expression in square brackets in each of these expressions and the term on the right hand side of eq. (12). In fact

$$
\left(\sigma_{\uparrow \uparrow}^{\mathrm{A}}+\sigma_{\uparrow \downarrow}^{\mathrm{A}}\right)-\left(\sigma_{\downarrow \downarrow}^{\mathrm{A}}+\sigma_{\downarrow \uparrow}^{\mathrm{A}}\right)=\sigma^{\mathrm{A}} P(\mathrm{~A})
$$

if

$$
\sigma_{\uparrow \downarrow}^{\mathrm{A}}=\sigma_{\downarrow \uparrow}^{\mathrm{A}} .
$$

The implications of eq. (18) may be illustrated by the following considerations: There are several processes which may contribute to the spin-flip probabilities of $\sigma_{\downarrow \uparrow}$ and $\sigma_{\uparrow \downarrow}$. These are:

1. The existence of components of $\boldsymbol{B}_{\boldsymbol{g}}$ perpendicular to the polarization direction as shown by the cross sections in eqs. (1)-(4);

2. Local distortion of the magnetization (particularly at surface imperfections) causing local $z$-axes not aligned with the external field, thus the neutron spin 
Number of neutrons of each spın state in each regıon under various conditıons.

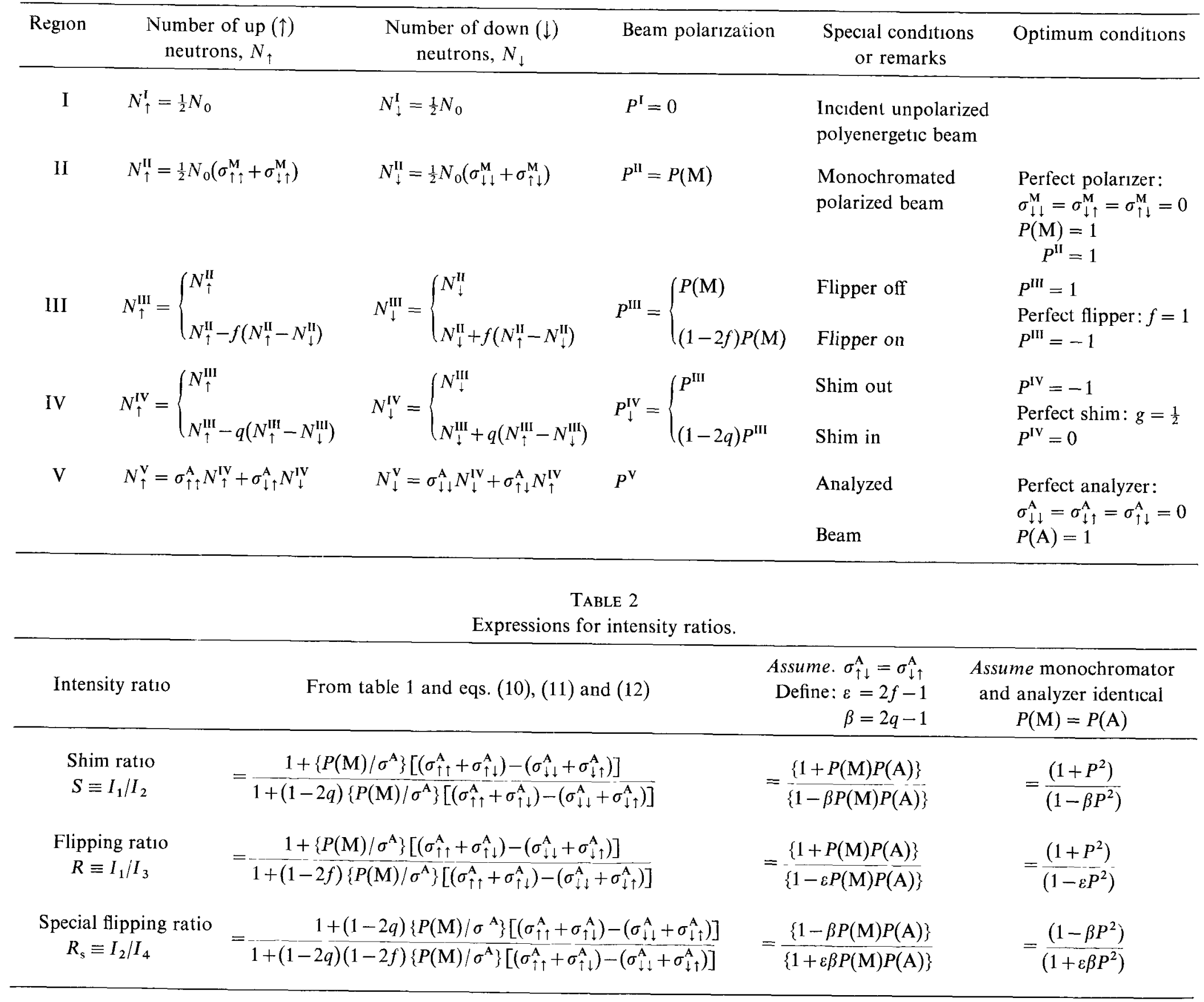


may be flipped in a scattering process in this local region; or

3. The neutron may precess about the local $z$-axis before or after a scattering event.

Our experience with the $\mathrm{Co}(8 \% \mathrm{Fe})$ monochromators and analyzers has been that the surface conditions are extremely important in seeking to eliminate these depolarizing spin-flip processes. Simple arguments then lead to the conclusion that $\sigma_{\uparrow \downarrow}=\sigma_{\downarrow \uparrow}$ if both the entrant and exit surfaces are identical as far as the three processes listed above are concerned. We will assume, therefore, that eq. (18) is valid. The modifications to the intensity ratios [eq. (16)] are shown in the third column of table 2 . We note, however, that the measurement of the three ratios of eq. (16) are not sufficient to determine the four unknown quantities $P(\mathrm{~A}), P(\mathrm{M})$, $\varepsilon(=2 f-1)$ and $\beta(=2 q-1)$. We could introduce a third crystal and measure the three ratios for every combination of the three crystals. This involves the inconvenience of changing the monochromator. Alternatively we can make an assumption about $P(\mathrm{~A})$ and $P(\mathrm{M})$. Some groups have used very thin analyzers and assumed $^{2}$ ) that $P(A)=1$. Since we have available crystals of the same size cut from the same boule and which show very similar double crystal rocking curves, we prefer to make the assumption that the polarizing efficiencies of the analyzer and monochromator are identical. Thus we assume

$$
P(\mathrm{~A})=P(\mathrm{M})=P
$$

and note that because of eq. (14), $P$ is the polarization of the beam scattered by the monochromator and incident on a sample crystal if the flipper is off and the shim is out. The simple expressions for the intensity ratios that result from this assumption are shown in the last column of table 2 . These expressions may be inverted to give

$$
\begin{gathered}
P^{2}=R_{\mathrm{s}}(S-1)(R-1) /\left(R-R_{\mathrm{s}}\right), \\
\varepsilon=\left(R-R_{\mathrm{s}} S\right) /\left\{R R_{\mathrm{s}}(S-1)\right\}, \\
\beta=R\left(1-R_{\mathrm{s}}\right) /\left\{R_{\mathrm{s}} S(R-1)\right\} .
\end{gathered}
$$

In summary, we can measure the polarization of the beam from the monochromator and the flipping efficiency with the Shim Method if we make just two assumptions. These are that the probability for spin-flip in the analyzer is the same for up to down as it is from down to up, and that the monochromator and analyzer have identical polarizing efficiencies because their other characteristics are similar. By measuring the special flipping ratio $R_{\mathrm{s}}$ we avoid the difficulty of assuming that the shim depolarizes the beam completely by measuring its depolarizing efficiency. We thus have a consistent set of measurements.

\section{Measurement of flipping ratio on sample crystals}

The measured flipping ratio on a test crystal is ${ }^{11}$ )

$$
R_{\mathrm{m}}=\{1+P P(\mathrm{~T})\} /\{1-\varepsilon P P(\mathrm{~T})\},
$$

in analogy with the previously defined $R$ (column 3 in table 2). The polarizing efficiency of the test crystal $P(\mathrm{~T})$ is defined according to eq. (12). The quantıty $R_{\mathrm{m}}$ is the ratio of two intensities, $I_{5}$ being that from the test crystal with flipper off and $I_{6}$ with flipper on. Eq. (23) is a fairly general expression for the measured flipping ratio in terms of the cross sections of the sample crystal. In order to equate it with eq. (7) we must make assumptions about the test crystal.

1. There is no extinction;

2. The sample is a simple ferromagnet so that there are no spin-flip processes due to $B_{g x}$ and $B_{g y}$ [eq. (4)] and we can write in accordance with eqs. (1) and (2)

$$
\sigma_{\uparrow \uparrow}^{\mathrm{T}} \propto(b+p)^{2} \text { and } \sigma_{\downarrow \downarrow}^{\mathrm{T}} \propto(b-p)^{2}
$$

3. The sample is saturated and the surfaces are highly polished to that there are no other spin-flup processes as discussed in section 3 and we can further assume

$$
\sigma_{\uparrow \downarrow}^{\mathrm{T}}=\sigma_{\downarrow \uparrow}^{\mathrm{T}}=0 \text {. }
$$

Solving eq. (23) for the polarizing efficiency of the test crystal $P(\mathrm{~T})$ gives

$$
P(\mathrm{~T})=\left(R_{\mathrm{m}}-1\right) /\left\{P\left(\varepsilon R_{\mathrm{m}}+1\right)\right\} .
$$

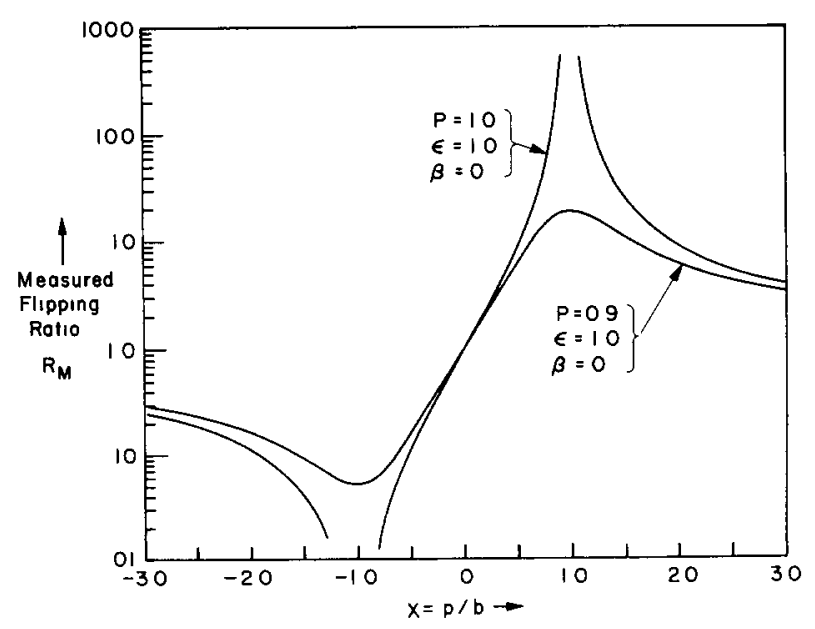

Fig 2. Plots of the measured flıpping ratıo $R_{\mathrm{In}}$ as a function of $X \equiv p / b$ for two combinations of the instrumental parameters $P, \varepsilon$ and $\beta$. 
Since

$$
P(\mathrm{~T}) \equiv\left\{(b+p)^{2}-(b-p)^{2}\right\} /\left\{(b+p)^{2}+(b-p)^{2}\right\},
$$

we have that $X$ is related to the measured polarizing efficiency by

$$
X \equiv p / b=\{1 / P(\mathrm{~T})\} \pm\left[\{1 / P(\mathrm{~T})\}^{2}-1\right]^{\frac{1}{2}} .
$$

Eqs. (26) and (28) show how the quantity of interest in the test crystal $p / b$, is related to the measured quantities. Plots of $R_{\mathrm{m}}$ against $X$ are shown in fig. 2 for two values of the incident beam polarization $(P)$. The plots are on a semi-log scale because of the rapid change of $R_{\mathrm{m}}$ as $|X|$ approaches unity. It may be noted that if $\varepsilon=1$ then

$$
R(X)=1 / R(-X)
$$

\section{Propagation of statistical errors}

The statistıcal errors in the experımental determınation of $X$ arise from the measurement of the six intensities $I_{1}, I_{2} \ldots I_{6}$. We wish, therefore, to calculate the error in $X$ caused by the statistical errors in the six measured intensities.

The usual approach to error calculations is to use the first term in a Taylor expansion,

$$
\Delta X=[\mathrm{d} X / \mathrm{d} P(\mathrm{~T})]_{X_{0}} \Delta P(\mathrm{~T}) .
$$

The validity of this method depends (at least) on the existence of the derivative at $X_{0}$. From eq. (28) we see that none of the derivatives of $X$ with respect to $P(\mathrm{~T})$ exists at $P(\mathrm{~T})= \pm 1$ so that a Taylor expansion in the

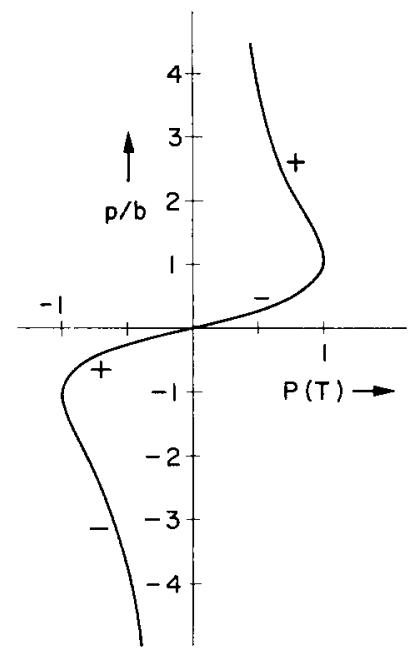

Fig 3 A plot of the polarizıng efficiency $P(\mathrm{~T})$ of a test crystal as a function of the ratio of the magnetic scattering length $p$ to the nuclear scattering length $b$ The $(+)$ and $(-)$ signs on the curve indicate the two branches of eq (28)

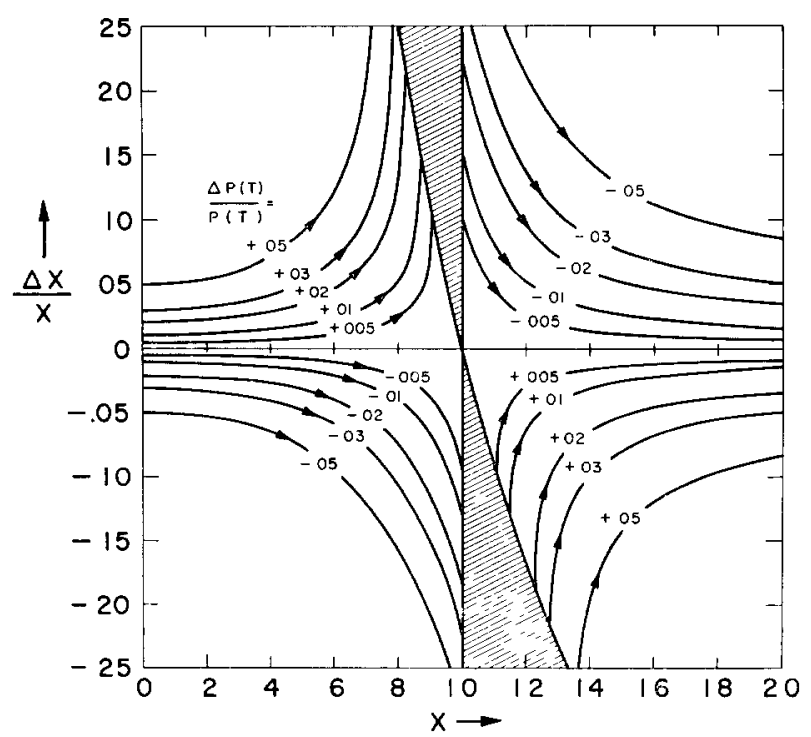

Fig 4. Because of the nonlinear relation between $X$ and $P(\mathrm{~T})$, this figure must be used in the calculation of $\Delta X / X$ for a given measured statıstical error $A P(\mathrm{~T})$ in $P(\mathrm{~T})$ The shaded regions are physically inaccessible since the polarızıng efficiency of the sample can never be greater than 1

neighborhood of \pm 1 is invalid. We take the position, therefore, that $\Delta X$ can be calculated from $\Delta P(\mathrm{~T})$ according to

$X+\Delta X=\{P(\mathrm{~T})+\Delta P(\mathrm{~T})\}^{-1} \pm\left\{[P(\mathrm{~T})+\Delta P(\mathrm{~T})]^{-2}-1\right\}^{\frac{1}{2}}$.

$X$ is given in terms of $P(\mathrm{~T})$ by eq. (28). The relation between $p / b$ and $P(\mathrm{~T})$ is shown in fig. 3, which illustrates the obvious, but very important, physical limitation that $-1 \leqq P(\mathrm{~T}) \leqq+1$. The signs on various parts of the curve refer to the sign in front of the radical in eq. (28). With the help of eqs. (28) and (31) curves of $\triangle X / X$ against $X$ were calculated for various values of $\triangle P(\mathrm{~T}) / P(\mathrm{~T})$ and are shown in fig. 4. The arrowheads indicate the direction of increasing $X$ for a particular value of $\Delta P(\mathrm{~T}) / P(\mathrm{~T})$. The two shaded portions are a consequence of the physical limitation we have already noted when it is applied to eq. (31). In this case the limitation is expressed by the curve $\Delta X / X=(1 / X)-1$. In general, for a fixed value of $\Delta P(\mathrm{~T}) / P(\mathrm{~T})$, we see that $\Delta X / X$ rises sharply as $X$ approaches 1 . We also note that the curves are asymmetric in $\Delta X / X$ for a given value of $X$ and magnitude of $\Delta P(\mathrm{~T}) / P(\mathrm{~T})$; for example If $X=0.8$ and $\Delta P(\mathrm{~T}) / P(\mathrm{~T})= \pm 0.02$ then $\Delta X / X=+0.13$ or -0.075 For positive values of $\Delta P(\mathrm{~T}) / P(\mathrm{~T})$ then $\Delta X / X$ is sharply peaked as $X$ increases from 0 to 1 so that, for example, $\Delta X / X$ at $X=0.95$ is less than $\Delta X / X$ at $X=0.90$ for $\Delta P(\mathrm{~T}) / P(\mathrm{~T}) \geqq+0.005$. We emphasize 
TABLE 3

Expressions for calculation of $\Delta P(\mathrm{~T})$.

$$
\Delta P(\mathrm{~T})= \pm\left[\left(A_{1}^{2} / I_{1}\right)+\left(A_{2}^{2} / I_{2}\right)+\left(A_{3}^{2} / I_{3}\right)+\left(A_{4}^{2} / I_{4}\right)+\left(A_{5}^{2} / I_{5}\right)+\left(A_{6}^{2} / I_{6}\right)\right]^{\frac{1}{2}}
$$

$$
\begin{aligned}
& \left|A_{1}\right|=\left[\left(1+P^{2}\right) /\left\{2 P^{2}(1+\varepsilon)(1+\beta)\right\}\right]\left[P(\mathrm{~T})\left\{(1-\varepsilon)+\beta\left(1-\varepsilon P^{2}\right)\right\}-2 \varepsilon P\{P(\mathrm{~T})\}^{2}\right] \\
& \left|A_{2}\right|=\left[\varepsilon\left(1-\beta P^{2}\right) /\left\{2 P^{2}(1+\varepsilon)(1+\beta)\right\}\right]\left[P(\mathrm{~T})\left\{1-P^{2}\right\}+2 P\{P(\mathrm{~T})\}^{2}\right] \\
& \left|A_{3}\right|=\left[\left(1-\varepsilon P^{2}\right) /\left\{2 P^{2}(1+\varepsilon)(1+\beta)\right\}\right]\left[P(\mathrm{~T})\left\{2+\beta\left(1+P^{2}\right)\right\}+2 P\{P(\mathrm{~T})\}^{2}\right] \\
& \left|A_{4}\right|=\left[\left(1+\varepsilon \beta P^{2}\right) /\left\{2 P^{2}(1+\varepsilon)(1+\beta)\right\}\right]\left[P(\mathrm{~T})\left\{1-P^{2}\right\}+2 P\{P(\mathrm{~T})\}^{2}\right] \\
& \left|A_{5}\right|=[P(\mathrm{~T})]^{2}\left[\left(1+X^{2}+2 P X\right)\left(1+X^{2}-2 \varepsilon P X\right) /\left\{4 P X^{2}(1+\varepsilon)\right\}\right] \\
& \left|A_{6}\right|=\left|A_{5}\right|
\end{aligned}
$$

again that this behavior is caused by the fact that it is physically impossible for a crystal to have a polarizing efficiency $P(\mathrm{~T})$ greater than 1 . The mirror images of these plots occur for the negative values of $X$.

In order to calculate now the error in $P(\mathrm{~T})$ due to errors in $I_{1}$ through $I_{6}$ we regard $P(\mathrm{~T})$ to be a function of $P, \varepsilon$ and $R_{\mathrm{m}}$ as expressed by eq. (26). We further regard $P$ and $\varepsilon$ as functions of the experimental intensity ratios $R, R_{\mathrm{s}}$ and $S$ (defined in terms of $I_{1}$ through $I_{4}$ in column 1 of table 2) as expressed by eqs. (20), (21) and (22). Since there are no further difficulties with the existence of derivatives we calculate $\triangle P(\mathrm{~T})$ due to, say, $I_{1}$ according to the first term in a Taylor expansion [eq. (30)]. Since the quantities $I_{1}$ through $I_{6}$ are statistically independent we write the expression for the total $\triangle P(\mathrm{~T})$ as

$$
\begin{aligned}
\Delta P(\mathrm{~T})= \pm\left[\left\{\left(\partial P(\mathrm{~T}) / \partial I_{1}\right) \delta I_{1}\right\}^{2}+\right. \\
\left.+\left\{\left(\partial P(\mathrm{~T}) / \partial I_{2}\right) \delta I_{2}\right\}^{2}+\ldots\right]^{\frac{1}{2}} .
\end{aligned}
$$

The partial derivatives are found by the "chain" rule from the relations between $P(\mathrm{~T})$ and $P, \varepsilon, R_{\mathrm{m}}$ as noted above. We also assume that

$$
\delta I_{\imath}=I_{\imath}^{\frac{1}{2}} .
$$

The expressions that result from this procedure are shown in table 3 where we have written for simplicity

$$
\begin{aligned}
\Delta P(\mathrm{~T})= \pm\left[\left(A_{1}^{2} / I_{1}\right)\right. & +\left(A_{2}^{2} / I_{2}\right)+\left(A_{3}^{2} / I_{3}\right)+ \\
& \left.+\left(A_{4}^{2} / I_{4}\right)+\left(A_{5}^{2} / I_{5}\right)+\left(A_{6}^{2} / I_{6}\right)\right]^{\frac{1}{2}} .
\end{aligned}
$$

We note in passing that the $I_{l}$ are corrected for background and that we include this in the computation of $\delta I_{l} / I_{\imath}$ as well as statistical errors in the monitor system. We can usually ensure that the monitor error is small so that we have written eq. (34) as a convenient approx1mation, bearing in mind, however, that $\delta I_{l} / I_{\imath}$ includes monitor and background errors in an actual calculation. The behavior of these coefficients $\left|A_{1}\right|$ with changing $X$ are plotted in fig. 5 for the two sets $P=\varepsilon=1, \beta=0$ and $P=0.9, \varepsilon=1, \beta=0$. For the perfect conditions we note the symmetry of the $A_{t}$ about $X=0$ and that $A_{3}=0$. If both $P$ and $\varepsilon$ are different from 1 then there is no symmetry about $X=0$. In our second case $A_{2}=A_{4}$ only because both $\beta=0$ and $\varepsilon=1$ as is evident from table 3 . Note that $\Delta P(\mathrm{~T})$ may be large near $X=1$ because of $A_{1}$, but also it may be large neal $X=0$ because of $A_{5}$ and $A_{6}$. For example if we make the somewhat unrealistic assumption (for convenience) that we have counted all intensities to an accuracy of about $4 \%$ then use of fig. 5 to find the $A_{1}$ (and table 3 ) shows that $\triangle P(\mathrm{~T}) \cong 0.05$ if either $X=0.1$ or 0.7 for $P=0.9, \varepsilon=1$ and $\beta=0$. In this case the percentage error $\Delta X / X$ is much larger for the case when $X=0.1$ than for $X=0.7$ as may be seen from fig. 4.

If an extensive experımental effort is directed toward form factor measurements it is worthwhile to prepare a series of figures of the type shown. The procedure is then as follows. Measurement of the intensities $I_{1}$ through $I_{4}$ yields values of $P, \varepsilon$ and $\beta$ according to eqs. (20), (21), (22) ... Measurement of $I_{5}$ and $I_{6}$ yields a value of $R_{\mathrm{m}}$ and fig. 2 is used to find the value of $X$ for the particular $P, \varepsilon$ and $\beta$. The polarizing efficiency is then easily calculated from eq. (27) or read off fig. 3. The $A_{\imath}$ are then read from fig. 5 and used to compute 
$\pm \Delta P(\mathrm{~T})$. The percentage error $\Delta X / X$ is then found from fig. 4. We will discuss further the experimental implications of calculations like those given in the example above in a later section.

\section{Systematic errors}

The systematic errors which arıse in an experimental measurement may be due to changing conditions in the experimental apparatus or to the lack of valıdity of some assumptions made about the measurement. In polarized neutron diffractometry an example of the for mer may be an increase in electronic noise when the rf flipper is on and an example of the latter may be that the sample is not a simple ferromagnet so that there is a spin-flup cross section. Such errors are often difficult to estimate and careful experiments must be devised to eliminate or attempt to measure them. We shall comment here on just four of the more obvious systematic errors.

\subsection{EXTINCTION}

In section 4 it was pointed out that in order to identify the measured flipping ratio with the simple cross section formula [eq. (7)] of section 2 it is necessary to assume that there is no extinction in the sample. There are a number of ways that this can be checked experimentally ${ }^{2}$ ) and it is our approach, in general, to reduce the sample dimensions until our checks indicate that there is no extinction. This may not always be practicable so we show how our previous expressions must be modified to include extinction effects.

For each material cross section the extinction coefficient is defined as the ratio of the observed intensity to that which should have been observed. Thus, for example,

$$
\sigma_{\uparrow \uparrow}^{\mathrm{T}}=E_{\uparrow \uparrow}^{\mathrm{T}} \sigma_{\uparrow \uparrow},
$$

where $\sigma_{\uparrow \uparrow}$ is the cross section for up-up scattering in the test sample material and $\sigma_{\uparrow \uparrow}^{\mathrm{T}}$ 1s the actual cross section for up-up scattering in the sample crystal. The extinction coefficient $E$ is initially linear with the cross section, i.e.

$$
E=1-\alpha \sigma,
$$

where $\alpha$ is a coefficient determıned by the sample geometry and mosaic spread, so that if the cross section is very small $E \rightarrow 1$. If we assume, again, that there are no spin-flip processes then the expressions for $R_{\mathrm{m}}$ and $P(\mathrm{~T})$ are modified as follows.

$$
\begin{aligned}
& R_{\mathrm{m}}=I_{5} / I_{6}=\left\{F(1+P)(1+X)^{2}+(1-P)(1-X)^{2}\right\} / \\
& \qquad /\left\{F(1-\varepsilon P)(1+X)^{2}+(1+\varepsilon P)(1-X)^{2}\right\}, \\
& P(\mathrm{~T})=\left\{2 X(1+F)-(1-F)\left(1+X^{2}\right)\right\} / \\
& \text { where } \quad /\left\{(1+F)\left(1+X^{2}\right)-2 X(1-F)\right\} \\
& \qquad F \equiv E_{\uparrow \uparrow}^{\mathrm{T}} / E_{\downarrow \downarrow}^{\mathrm{T}}=\left\{1-\alpha(1+X)^{2}\right\} /\left\{1-\alpha(1-X)^{2}\right\}
\end{aligned}
$$

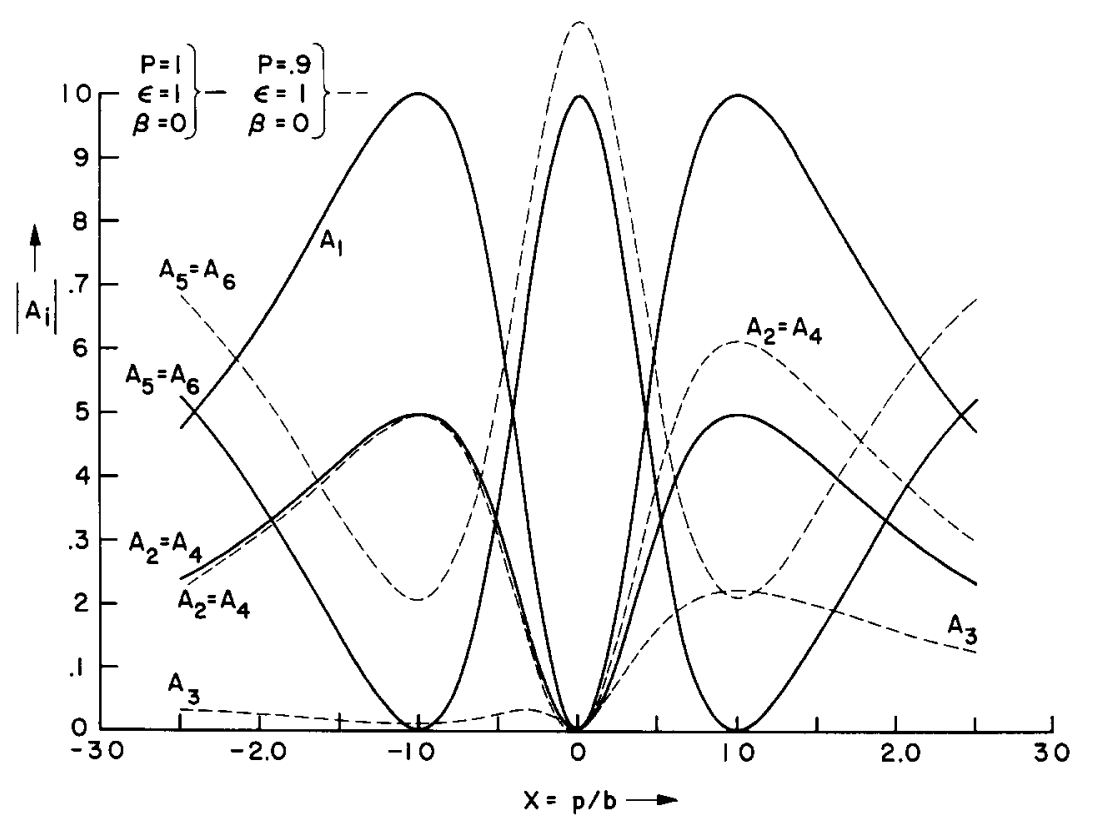

Fig 5 This figure shows the functional dependence of the error parameters $A$ of eq (34) on $p / b$ The plots are made for two choices of the instrumental parameters $P, \varepsilon$ and $\beta$. 
There is no reliable way of calculating $F$ and every effort must be made to avoid extinction in the sample. However, there are various methods of estimating it which with the formulas (37) and (38) can give an indication of the importance of extınction for varıous values of $X$.

\subsection{DRIFT OF FLIPPER EFFICIENCY WITH TIME}

Polarized neutron diffractometry frequently involves long counting times both because of the inherently low intensity avalable from the commonly used $\mathrm{Co}(8 \% \mathrm{Fe})$ polarizer and because the magnetıc scattering amplitudes are often small compared to the nuclear scattering amplitudes. Durıng long counting periods the flipping efficiency may change because of drift in the rf power supply. This necessitates periodic checks of the efficiency. The effect of this systematic error is most important if the magnetic and nuclear scattering amplitudes are nearly equal. If it is found that the efficiency changes substantially with time we suggest eliminating the flipper from the measurements on the test crystal. In its place we propose to use the shim, of which the depolarizing efficiency $\beta$ is already known. The propagation of the statistical errors follows the development of section 5 with the replacement of $\beta$ for $\varepsilon \mathrm{in}$ the expression for $R_{\mathrm{m}}$. The coefficients $A_{\mathrm{l}}$ of table 3 are replaced by new coefficients $B$, found by writing $\beta$ wherever there is an $\varepsilon$ and at the same time $\varepsilon$ wherever there is a $\beta$.

Thus

$$
\begin{array}{ll}
B_{1}=A_{1}(\beta \leftrightarrow \varepsilon) ; \quad & B_{4}=A_{4}(\beta \leftrightarrow \varepsilon) ; \\
B_{2}=A_{3}(\beta \leftrightarrow \varepsilon) ; & B_{5}=A_{5}(\beta \leftrightarrow \varepsilon) ; \\
B_{3}=A_{2}(\beta \leftrightarrow \varepsilon) ; & B_{6}=A_{6}(\beta \leftrightarrow \varepsilon) .
\end{array}
$$

The $B$ 's are always numerically larger than the $A$ 's, as is to be expected, so that this method is only preferable if the systematic error in $\varepsilon$ is large.

\subsection{SPIN-FLIP SCATTERING}

It is important to check the assumption that the sample is a simple ferromagnet. The method for doing this by polarization analysis of the scattered beam was indicated in section 2 .

\subsection{Depolarization IN THE SAMPLE}

This may arise if the sample is not saturated or if the surface conditions are not good, as already discussed in section 3. Depolarization can be checked for by interposing the magnetized sample between the monochromator and analyzer ${ }^{2}$ ). A procedure for dealing with this systematic error has been given by several authors ${ }^{12}$ ) and it involves the assumption that the depolarization in the specimen is uniform and is a function of the distance traversed by the beam through the test crystal. It has been our experience with $\mathrm{Co}(8 \% \mathrm{Fe})$ that the effect is a surface one and consequently we feel that the problem can be safely handled only by ensurıng that the sample is highly polished and magnetically saturated.

\section{Implications with regard to counting times}

In section 5 we have described the procedure for a rigorous calculation of the statistical error involved in the measurement of the ratio $p / b$. The calculation is rather complex and it is not easy to see through it in order to draw conclusions about how a given experıment should be performed. A practical question which often arises is how long to spend on each intensity measurement and a solution is frequently given after assuming that a fixed time is avallable for a given experiment. We take the position here rather that sufficient time is available to find $p / b$ to the desired accuracy. As an example we propose that each of the six terms in eq. (34) should contribute equally to the error $\Delta P(\mathrm{~T})$. If we denote a counting rate by $C_{t}$, then the total accumulated count in a time $t_{t}$ is

$$
I_{t}=C_{t} t_{i}
$$

For comparable contributions to the error $\triangle P(\mathrm{~T})$ from each term in eq. (34), we must have

$$
t_{\imath} / t_{1}=\left(C_{1} / C_{\imath}\right)\left(A_{d} / A_{1}\right)^{2}
$$

where the $A_{t}$ are given in table 3 and the ratios $C_{1} / C_{t}$ are given in table 4 . Note that the expressions for $C_{1} / C_{5}$ and $C_{1} / C_{6}$ contain the ratio of the cross sections of the sample and analyzer. We emphasize again that these are Bragg scattering cross sections and so contain crystal parameters like the volume and mosaic spread.

TABLE 4

Expressions for calculation of countıng times for equal contributions to the error $\triangle P(T)$.

$$
\begin{aligned}
& t_{1} / t_{1}=\left(C_{1} / C_{i}\right)\left(A_{1} / A_{1}\right)^{2} \\
& C_{1} / C_{2}=\left(1+P^{2}\right) /\left(1-\beta P^{2}\right) \\
& C_{1} / C_{3}=\left(1+P^{2}\right) /\left(1-\varepsilon P^{2}\right) \\
& C_{1} / C_{4}=\left(1+P^{2}\right) /\left(1+\varepsilon \beta P^{2}\right) \\
& C_{1} / C_{5}=\left\{\sigma^{\mathrm{A}}\left(1+P^{2}\right)\right\} /\left\{\sigma^{\mathrm{T}}[1+P P(\mathrm{~T})]\right\} \\
& C_{1} / C_{6}=\left\{\sigma^{\mathrm{A}}\left(1+P^{2}\right)\right\} /\left\{\sigma^{\mathrm{T}}[1-\varepsilon P P(\mathrm{~T})]\right\}
\end{aligned}
$$


The counting time ratios $t_{t} / t_{1}$ are plotted against $p / b$ in fig. $6 \mathrm{a}$ for $P=1.0$ and in fig. $6 \mathrm{~b}$ for $P=0.9$ with $\varepsilon=1$ and $\beta=0$ in both cases. For these values of $\varepsilon$ and $\beta, t_{2} / t_{1}=t_{4} / t_{1}$ as is evident from tables 3 and 4 . The main features of all these curves are that for $p / b= \pm 1, t_{t} / t_{1} \ll 1$; and that $t_{t} / t_{1} \rightarrow \infty$ as $p / b \rightarrow 0$. These characteristics are not unexpected in view of the behavior of the $A_{1}$ shown in fig. 5. A similar calculation can be made if the shim ratio method mentioned in $\S 6.2$ is used. The ratios of the counting times are, of course, always larger than when the flipper is used.

We conclude this section with a comment on the polarization of the beam incident on the test crystal. It is rather obvious that the further $P$ is from one, the

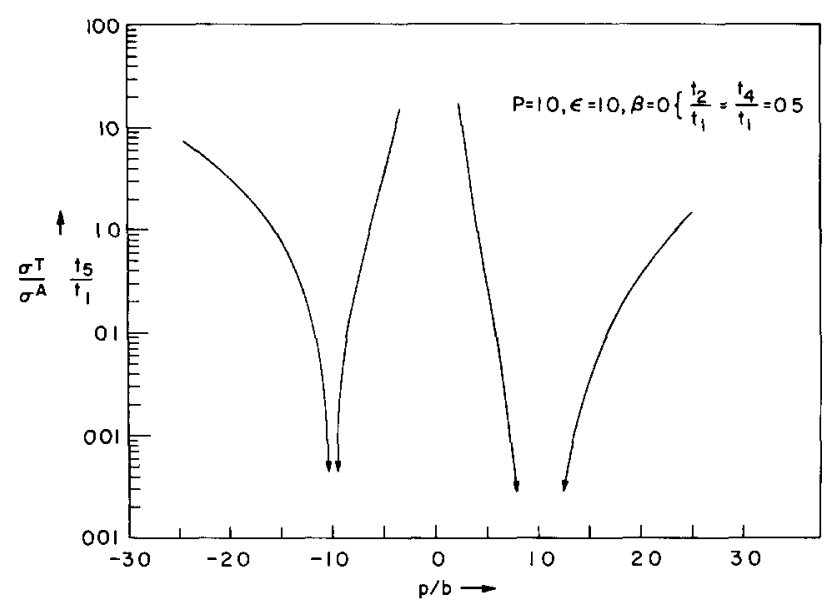

(a)

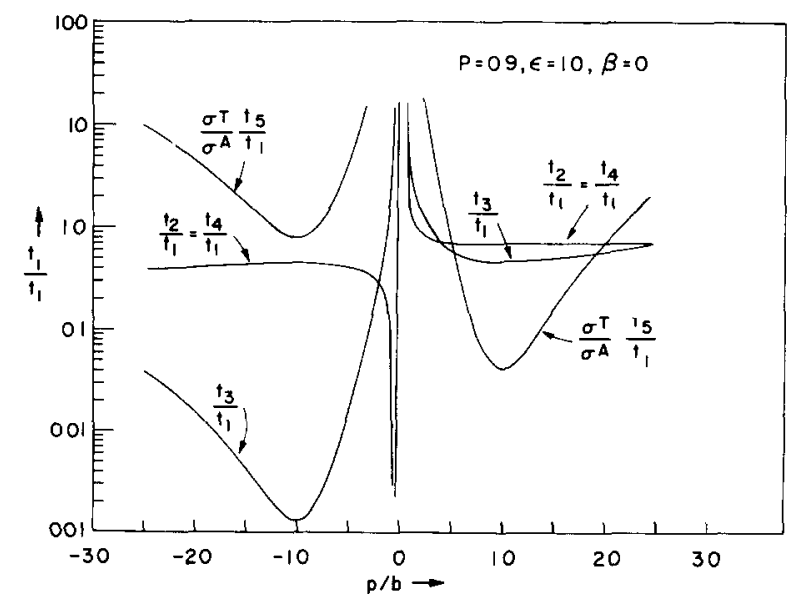

(b)

Fig. 6. Plots of the relative counting tıme ratios $t_{i} / t_{1}$ as a function of $p / b$ necessary to obtain equal contributions (from each of the six indices) to the statıstical error $\triangle P(T)$ in the polarızing efficiency of the sample crystal, for (a) $P=10, \varepsilon=1.0, \beta=0$; (b) $P=09, \varepsilon=1.0, \beta=0$. larger the correction which must be applied to the measured flipping ratio $R_{\mathrm{m}}$ in order to obtain $p / b$, particularly when $p / b \rightarrow 1$. A corollary is that any uncertainty in $P$ becomes more serious in determining $p / b$ the more $P$ differs from 1 . This is quite evident from the plot of $R_{\mathrm{m}}$ against $p / b$ in fig. 2 , where it is seen that when $p / b=1, R_{\mathrm{m}}$ changes from $\infty$ when $P=1$ to 19 when $P=0.9$. There is no doubt, therefore, that for accurate form factor determinations in the vicinity of $p / b=1$ every effort should be directed toward obtaining a beam which is as close to $100 \%$ polarized as possible.

\section{Summary and conclusions}

In this paper we have shown how to calculate the statistical errors involved in measuring magnetıc form factors using polarized neutrons. The discussion began by reviewing the interference cross section for Bragg scattering of polarized neutrons in order that the usual assumptions implied by the method would be clear. Emphasis was placed on the assumptions required to arrive at the familiar expression for the flipping ratio in simple ferromagnets [eq. (7)]. It was pointed out that the assumption of a simple ferromagnet (no spin-flıp scattering) can be checked by polarization analysis of the scattered beam.

In section 3 we developed expressions for the various intensity ratios measured when using the shim ratio method for determining the polarization of the beam and the flipper efficiency. Emphasis was again placed on the assumptions required to arrive at the commonly used expressions. It was pointed out that an additional measurement (flipper on, shim in) leads to the determination of the depolarızing efficiency of the shim and consequently provides a consistent set of measurements. This work was extended in section 4 to the measurement of the flipping ratio from a test crystal. The additional assumptions required to interpret the results in terms of the material cross sections or scattering lengths $(p, b)$ were noted.

In section 5 we derived expressions for the propagation of the statistical errors involved in measuring the six intensities necessary for a determination of $p / b$. A procedure for finding the error in $p / b$ from a series of plots was outlined and a numerical example was given.

In section 6 we discussed some of the systematic errors which can arise in polarized neutron diffractometry. There appears to be no safe way of correcting for extinction in a test crystal, and we concluded that for an accurate determination of $p / b$ the extinction must be negligible.

We suggested that if the rf power supply causes drift 
In the flipper efficiency over long counting times then it may be preferable to avold this systematic error by using a shım instead, particularly when $p / b$ is close to 1 .

Although procedures for correcting for depolarization in the specimen have been suggested by others we feel that accurate measurements of $p / b$ can be made only if this source of error is eliminated. This can be done by ensuring that the sample is highly polished and magnetically saturated.

There are, of course, several other difficulties which can arise in polarized neutron measurements, notably multıple Bragg scattering. The treatment of this difficulty (rotation about the scattering vector) and others have been described elsewhere.

In section 7 we discussed some of the experimental implications of the error analysis. We showed how to calculate the counting times required if it is desired to have equal error contributions from the six intensity measurements. The principal results were that large counting time ratios are necessary when $p / b \rightarrow 0$ and that small time ratios are involved if $p / b \rightarrow \pm 1$. We draw attention here, however, to the fact that the calculations were of counting tıme ratios and that the effects of fig. $4[\Delta X / X$ vs $X$ for values of $\Delta P(\mathrm{~T}) / P(\mathrm{~T})]$ were not included.

\section{References}

1) R. Nathans, M T. Pigott and C. G Shull, J Phys Chem Solids 6 (1958) 38 .

2) R. Nathans, C. G. Shull, G. Shirane and A Andresen, J Phys Chem Solids 10 (1959) 138

3) R. Nathans and A Paolettı, Phys. Rev Letters 2 (1959) 254

4) R Nathans and S J. Pickart, Phys Rev 123 (1961) 1163

5) C G. Shull and Y. Yamada, J. Phys Soc. Japan 17, Suppl. B-III (1962) 1

6) R Nathans, S J Pickart, H. A Alperin and P. J Brown, Phys Rev 136 (1964) A1641

7) Ref ${ }^{10}$ ) or, for example, P. deGennes in Magnetssm 3 (ed. G T Rado and H Suhl; Academic Press, N Y, 1963) or H Kendrick, Ph D Thesis (The Unıversity of Michıgan, 1968), unpublished.

8) A W. Overhauser, Bull. Am Phys Soc (2), 7 (1962) 241

9) For example, A Arrott in Magnetism 2B (ed G T Rado and H Suhl; Academic Press, N Y, 1966).

10) O. Halpern and M Johnson, Phys Rev 55 (1938) 898.

11) R. Nathans and S. J. Pickart in Magnetism 3 (ed G. T Rado and H. Suhl; Academic Press, N Y, 1963)

12) For example, P J Brown and J B. Forsyth, Brit J Appl. Phys 15 (1964) 1529. 